\title{
Design of the ECE diagnostic at Wendelstein 7-X
}

\author{
S. Schmuck, H.-J. Hartfuss, M. Hirsch, T. Stange
}

December 19, 2008

Max-Planck-Institut für Plasmaphysik, Teilinstitut Greifswald, EURATOM Association, Wendelsteinstraße 1, D-17491 Greifswald

abstract: The Electron Cyclotron Emission diagnostic will be a main tool for the measurement of the electron temperature profile in the stellarator Wendelstein 7-X presently under construction in Greifswald, Germany. Design criteria and constraints for the ECE diagnostic are discussed. The resulting set-up is introduced with respect to the line of sight and the in-vessel optics. The 32-channel heterodyne radiometer system already used at the predecessor experiment Wendelstein 7-AS is adapted to the conditions at Wendelstein 7-X.

keywords: ECE, heterodyne principle, Gaussian beam telescope

\section{Introduction}

The optimized modular steady state stellarator Wendelstein $7-\mathrm{X}$ is presently under construction in Greifswald. Wendelstein 7-X has the avaraged major radius of $5.5 \mathrm{~m}$ and the avaraged minor radius of $0.53 \mathrm{~m}$. The envisaged maximum electron temperature is about $10 \mathrm{keV}$ and the highest expected electron density is some $10^{20} \mathrm{~m}^{-3}$. For densities up to $1.2 \cdot 10^{20} \mathrm{~m}^{-3}$ the electron cyclotron resonance heating $(\mathrm{ECRH})$ is suited for depositing energy into the plasma using microwaves with the frequency of $140 \mathrm{GHz}$ and X-mode polarization. At Wendelstein 7-X the ECRH will be installed with a maximum power of 10 Megawatt. The physical process behind the ECRH is inverse with respect to the mechanism provoking the electron cyclotron emission (ECE). There the plasma electrons emit microwaves due to the acceleration by the Lorentz force involving the magnetic field. For the standard configuration of Wendelstein $7-\mathrm{X}$ the magnetic field is $2.5 \mathrm{~T}$ near the magnetic axis. The corresponding fundamtenal ECE spectrum is in the range of $70 \mathrm{GHz}$. Since the high aspect ratio of Wendelstein 7-X the magnetic field variation across the minor radius is sufficiently small such that the spectral range of the second harmonic (around $140 \mathrm{GHz}$ ) does not overlap with the fundamental ECE spectrum. Only the second harmonic spectrum overcomes the cut-off frequency up to the density of $1.2 \cdot 10^{20} \mathrm{~m}^{-3}$. Therefore the second harmonic ECE spectrum is suited to deliver information of plasma parameters in particular the electron temperature.

For the expected electron density and temperature range at Wendelstein $7-\mathrm{X}$ and affected by the relativistic line broadening, the optical thickness for the X2-mode is sufficiently high to ensure radiation at the blackbody level [1]. Therefore the ECE diagnostic can determine the electron temperature profile from the intensity and the frequency of the ECE emitted by the plasma. Besides the capability of a high spatial resolution the ECE diagnostic also offers a high temporal resolution which altogether enables the measurement of temperature fluctuations. Thus for Wendelstein 7 -X the ECE diagnostic will be a main electron temperature diagnostic $[2,6]$.

One major component of the ECE diagnostic is a multichannel radiometer system built and oper- 
ated already at Wendelstein 7-AS which was the predecessor of Wendelstein 7-X. The radiometer system was developed for the second harmonic mode (X2-mode) in the spectral range from $126 \mathrm{GHz}$ to $160 \mathrm{GHz}$. By making use of the heterodyne principle this spectrum is down-converted to $4 \mathrm{GHz}$ to $40 \mathrm{GHz}$ and subsequently transmitted to a filterbank with 32 channels. Thus the temperature profile is measured at 32 locations. In this paper, first of all the constraints for the ECE diagnostic design at Wendelstein 7-X are given. The resulting design is presented including the in-vessel optics. Affecting the performance of the ECE diagnostic the parameters along the sight line are discussed. The procedure ultimately aims on a fully probabilistic analysis of the temperature measurement including error sources. Furthermore for application at Wendelstein 7-X the spatial resolution of the radiometer system has to be readapted. This requires the information about the magnetic field along the ECE sight line. First steps of optimization for the spatial resolution of the radiometer system will be presented and discussed.

\section{Design of the ECE diagnostic}

In this section general physical constraints for ECE are formulated which determine engineering aspects of the ECE diagnostic at Wendelstein 7-X. Second the individual components of the ECE diagnostic and their design criteria are described.

\subsection{Boundary conditions and constraints}

The standard ECE diagnostic requires a magnetic field increasing monotonically with distance from the antenna. In a tokamak the standard ECE diagnostic observes the plasma from the outer torus side fulfilling the demands of ECE for the magnetic field to decrease along the sight line to the observer. For stellarators in general and also for Wendelstein 7-X the magnetic field variation can be much more complicated and can be both increasing and decreasing along the line of sight. Fortunately sight lines exist with a tokamak-like field variation which have been chosen for the standard ECE diagnostic as well as for the ECRH heating systems of Wendelstein 7-X providing most favourable conditions for EC emission and absorption.

For the standard configuration of Wendelstein $7-\mathrm{X}$ the magnitude of the magnetic field is about $2.5 \mathrm{~T}$ on the magnetic axis. For the gyromotion of the electrons, the fundamental mode is in the range of $70 \mathrm{GHz}$ and the second harmonic mode (X2-mode) is in the range of $140 \mathrm{GHz}$. Considering the magnetic field variation inside the last closed flux surface the spectrum of the X2-mode covers the range from $126 \mathrm{GHz}$ to $162 \mathrm{GHz}$.

At Wendelstein 7-X the main heating source will be the ECRH available at $140 \mathrm{GHz}$. In order to protect the ECE diagnostic from power overload, notch filters are implemented in the signal line blocking a narrow spectral range around the frequency of $140 \mathrm{GHz}$. Thus ECE is not able to measure at the specific location corresponding to the blocked frequency. If this frequency is related to the magnetic axis, which offen is the position with the highest temperature, this value can not be measured directly. To avoid this disadvantage, the ECE sight line has been chosen such that the magnitude of the magnetic field at the magnetic axis deviates from $2.5 \mathrm{~T}$ sufficiently.

Recent work has shown that the simultaneous investigation of the plasma from the high-field and the low-field side along the same sight line can provide information about the high energy tail of the electron distribution function $[1,3]$. For this purpose an additional ECE antenna is planned to be implemented at the inboard side of Wendelstein 7-X. Thus a side condition is, that the sight line crosses the inner torus vessel wall in a region where a high-field side antenna system can be realized from the engineering point of view. 


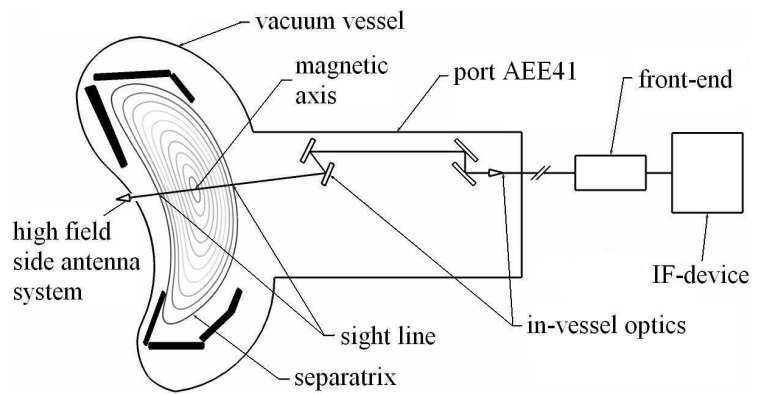

Figure 1: Cross section of Wendelstein 7-X at ECE diagnostic port AEE41 at toroidal angle of 6.1 degree. The main components of the ECE diagnostic are the in-vessel optics determining the sight line and the multichannel radiometer system consisting of the front-end and the intermediate-frequency-device (IF-device). Oversized waveguides with a length of $20 \mathrm{~m}$ guide the microwaves to the front-end placed outside the torus hall. A thin mica window is planed to be installed. The position of the high-field side antenna system is sketched, however, the corresponding transmission line has been omitted. The plasma poloidal cross section is bean-shaped.

\subsection{Overall design}

The current design of the ECE diagnostic shown in figure 1 is compatible with the formulated constraints. The ECE diagnostic is placed at the toroidal angle of about 6.1 degree in the top of the port AEE41 that is shared with spectroscopic diagnostics.

A high-field side antenna system probing the fast electron species can be realized but is beyond the scope of this paper.

Starting from the plasma the low-field side antenna system consists of the following components: The in-vessel optics resembles a Gaussian beam telescope which together with the corrugated feed horn collects the microwave spectrum emitted by the plasma. A vacuum window made of mica (aperture $4 \mathrm{~mm}$ ) is planned to be installed, as it was already successfully used at Wendelstein 7AS. It allows the required broad spectral transmission in the microwave range. With oversized waveguides of about $20 \mathrm{~m}$ length the microwaves are guided to the radiometer outside the torus hall. The multichannel radiometer system makes use of the heterodyne principle [6]; the spectrum is down-converted to a lower frequency range by the front-end providing a better signal handling. The intermediate-frequency-device (IF-device) is fed with the shifted spectrum which is finally covered by a filterbank with 32 channels, each followed by a detection diode. In the next paragraphs specific topics of the main components will be discussed.

\subsection{Sight line characteristics}

In general the spatial resolution of the ECE diagnostic is limited by the relativistic line broadening, absorption and reemission processes. In order to reach this limit, other unwanted effects like refraction and Doppler shift have to be minimized. For that purpose the required quantities have to be stated and evaluated.

The propagation of the microwaves and thus the probing position in the plasma is affected by refraction effects. Refraction plays a role if the sight line is not orientated parallel with respect to the density gradient. In a magnetically confined plasma the density gradient has the same direction as the gradient of the flux surfaces. Thus the angle between these vectors is a measure for the level of refraction effects.

For the ECE diagnostic, the Doppler effect is a preventable line broadening mechanism. The effect can be decreased if the angle between the sight line and the magnetic field approaches 90 degree.

The IF-device has to be readapted for conditions at Wendelstein 7-X. Since the magnetic field links the frequency of the emitted microwaves with the radial position, the spatial resolution of the 

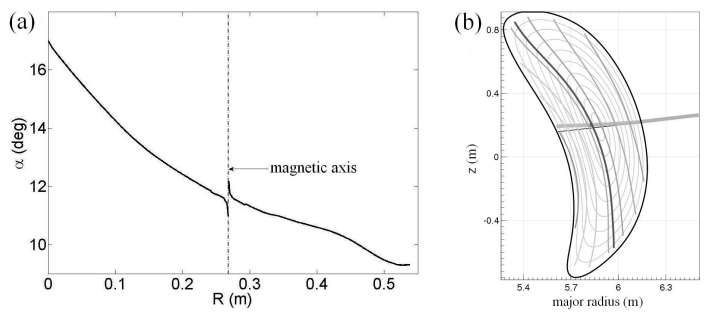

Figure 2: Refraction for ECE diagnostic at Wendelstein 7-X. (a) Angle $\alpha$ between gradient of flux surfaces and ECE sight line for vacuum case. The position $\mathrm{R}=0 \mathrm{~m}$ corresponds to the crossing of the sight line with the separatrix on the high-field side (inner torus side). The crossing of the separatrix on the low-field side (outer torus side) is at the position $\mathrm{R}=0.53 \mathrm{~m}$. The sight line crosses the magnetic axis (dash-dotted line) at $\mathrm{R}=0.267 \mathrm{~m}$. A small value of $\alpha$ corresponding to negligible refraction is desireable for a good spatial resolution. The angle varies from 17 degree to 9 degree. At the magnetic axis the gradient is not defined causing a different left-hand limit with respect to the right-hand limit of $\alpha$. (b) Ray-tracing results for line of sight for frequency of $140 \mathrm{GHz}$ and flat electron density profile with central density of $8 \cdot 10^{19} \mathrm{~m}^{-3}$. The modified path (thick gray line) deviates from the straight vacuum way (thin black line) inside the last closed flux surface (outermost closed line). The constant magnitude of the magnetic field of $2.5 \mathrm{~T}$ (thick vertical line) is the cold resonance for the chosen frequency of $140 \mathrm{GHz}$. At the cold resonance the refraction causes a small deviation of about $2 \mathrm{~cm}$ in the height $\mathrm{z}$ and $1 \mathrm{~cm}$ along the major radius.

ECE diagnostic can be changed by the choice of the filter bandwidth. This procedure requires the knowledge of the magnetic field along the sight line. The finally chosen filter bandwidth should be small enough that the spatial resolution is determined solely by the ECE emission, the antenna and sight line parameters and not by the filterbank. On the other hand the signal-to-noise ratio increases with the bandwidth.

The mentioned issues above were examined with a software which uses an interface to already existing magnetic field calculation codes. The gradient of the flux surfaces and the vectorial magnetic field are gained for points along a given sight line for the vacuum case and for the standard magnetic configuration of Wendelstein 7-X.

The angle between the sight line and the gradient of the flux surfaces is given in figure 2a. On the high-field side the angle is at the highest value with approximately 17 degree. With the ray-tracing tool [1] the refraction was calculated for the frequency of $140 \mathrm{GHz}$ and a flat electron density profile with central density of $8 \cdot 10^{19} \mathrm{~m}^{-3}$. The figure $2 \mathrm{~b}$ shows the deviation between the line of sight for vacuum case and for non-vacuum case. Even for the chosen high density the deviation from the straight unrefracted line is $2 \mathrm{~cm}$ in vertical direction within the direction of the flux surface.

The angle between sight line and the magnetic field is shown in figure 3a. The angle changes
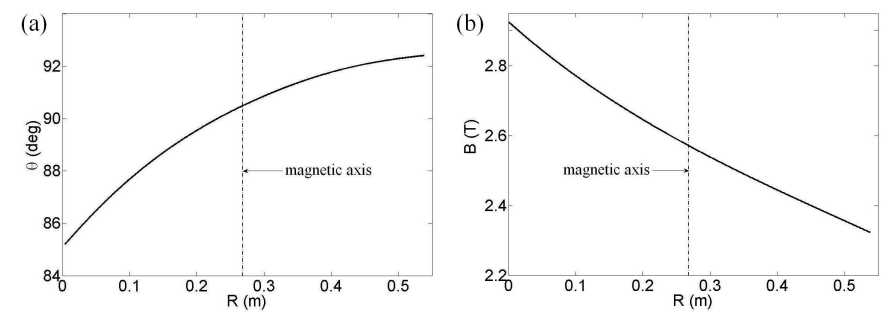

Figure 3: Different parameters along the chosen ECE sight line inside last closed flux surface. The coordinate R is explained in figure 2 (a) Angle $\theta$ between magnetic field and ECE sight line determining the Doppler effect. The angle is close to 90 degree where Doppler broadening is minimized. On the magnetic axis $\theta$ is about 90 degree. (b) Magnitude of magnetic field $B$. The magnetic field is about $2.9 \mathrm{~T}$ at the high-field side, $2.55 \mathrm{~T}$ on the magnetic axis and $2.3 \mathrm{~T}$ at the low-field side. 
(a)

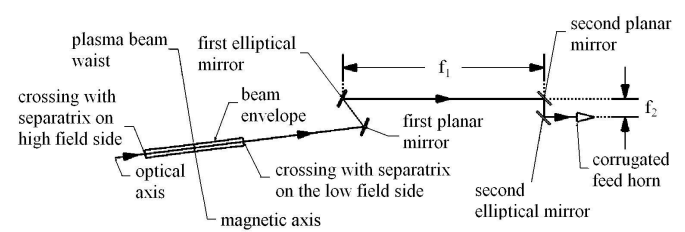

(b)

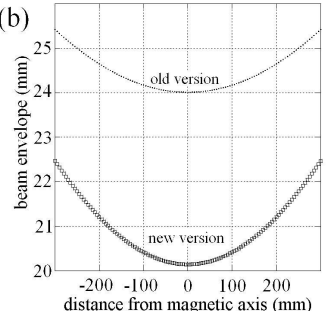

Figure 4: Design of in-vessel optics for ECE diagnostic on low-field side at Wendelstein 7-X. (a) Realization of in-vessel optics. The application of a Gaussian beam telescope ensures a wavelength independing beam envelope inside the last closed flux surface. Following the optical axis the two elliptical mirrors are placed at a distance of $1.25 \mathrm{~m}$ corresponding to the sum of the focal lengths of $\mathrm{f}_{1}=1.1 \mathrm{~m}$ and $\mathrm{f}_{2} .=0.15 \mathrm{~m}$. The corrugated feed horn has a beam waist of $3.2 \mathrm{~mm}$. (b) Dimension of beam envelope inside the last closed flux surface for two combinations of $\mathrm{f}_{1}$ and $\mathrm{f}_{2}$. The previous version covers a bigger plasma volume than the optimized version.

slightly but stays close to 90 degree. Thus the Doppler effect will not be the main line broadening mechanism for the ECE sight line.

In figure $3 \mathrm{~b}$ the magnitude of the magnetic field is shown for the ECE diagnostic sight line. The magnetic field is monotonically decreasing from $2.9 \mathrm{~T}$ at the high-field side to $2.3 \mathrm{~T}$ at the low-field side.

\section{$2.4 \quad$ In-vessel optics}

For the ECE diagnostic the sight line respectively the antenna characteristics are determined by the in-vessel optics. The radiative heat flux is about $100 \mathrm{~kW} / \mathrm{m}^{2}$ on the vessel walls for Wendelstein 7$\mathrm{X}$. Therefore in-vessel components including the stainless steel optics of the ECE diagnostic have to be actively cooled. Cooling aspects are considered in the actual design of the ECE diagnostic but are beyond the scope of this contribution.

To ensure that the beam dimension and thus the size of the observed plasma volume is independent of the wavelength at Wendelstein 7-X a Gaussian beam telescope is used as viewing optics. The applied concept of the Gaussian beam telescope includes two elliptical mirrors placed at the distance which equals the sum of their focal lengths as shown in figure $4 \mathrm{a}$. The mechanical components will be made of stainless steel with reduced fraction of cobalt avoiding an activation of the material by neutrons.
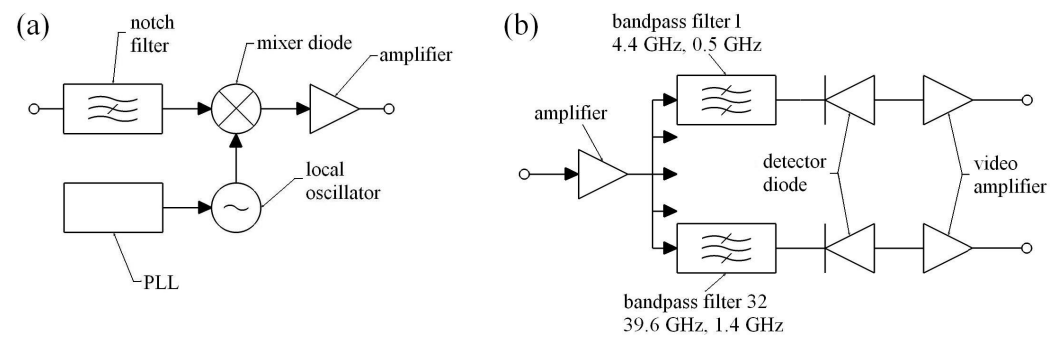

Figure 5: Multichannel radiometer system of ECE diagnostic at Wendelstein 7-X. The radiometer system consists of the front-end and the IF-device. (a) Front-end block diagramm. The spectrum passes the notch filter blocking the frequency of $140 \mathrm{GHz}$. The mixer multiplies the spectrum with the local oscillator signal of the frequency of $122.12 \mathrm{GHz}$ stabilized by a phase-locked loop (PLL). The generated intermediate frequencies (IF) are amplified. (b) IF-device block diagramm. The down-converted spectrum is amplified and splitted by power dividers ending up in 32 channels. Each channel has a bandpass filter which transmittes a narrow spectral range detected by a diode. The video amplifier amplifies the diode output subsequently sent to the data aquisition. 
The corrugated feed horn with the beam waist of $3.2 \mathrm{~mm}$ and the focal lengths of the elliptic mirrors determine the beam envelope inside the last closed flux surface. The dimension of the beam envelope in the confined plasma is shown in figure $4 \mathrm{~b}$ for two different design versions. The optimized version achieves a beam waist of $20 \mathrm{~mm}$ at the magnetic axis. The beam waist variation is small $(2 \mathrm{~mm})$ allowing a nearly constant beam envelope all along the sight line.

\subsection{Multichannel radiometer system}

After a short description of the multichannel radiometer system, first steps of the preparation for the application at Wendelstein $7-\mathrm{X}$ are presented.

The multichannel radiometer consisting of the front-end and the IF-device was developed for Wendelstein 7 -AS conditions taking advantage of the heterodyne principle $[4,5,6]$. A detailed description of the two components can be found in [4], [5]. The main features are sketched in figure 5. The front-end converts the spectrum of the X2-mode $(126 \mathrm{GHz}-162 \mathrm{GHz})$ to the intermediate frequency (IF) range from $4 \mathrm{GHz}$ to $40 \mathrm{GHz}$. This is achieved by the mixer diode multiplying the spectrum with the local oscillator signal of frequency of $122.12 \mathrm{GHz}$ stabilized by a phase-locked loop (PLL). The difference frequencies generated by the mixer diode are amplified and analyzed by the IF-device. The spectrum is manifolded by power dividers giving 32 branches. Every branch is equipped with a bandpass filter transmitting a narrow spectral range, a diode for detection and a video amplifier. The center frequency of the bandpass filter is in the range from $4.4 \mathrm{GHz}$ to $39.6 \mathrm{GHz}$. The filter bandwidth varies from $0.25 \mathrm{GHz}$ to $1.4 \mathrm{GHz}$ adapted to the magnetic field variation for the sight line at Wendelstein 7 -AS.
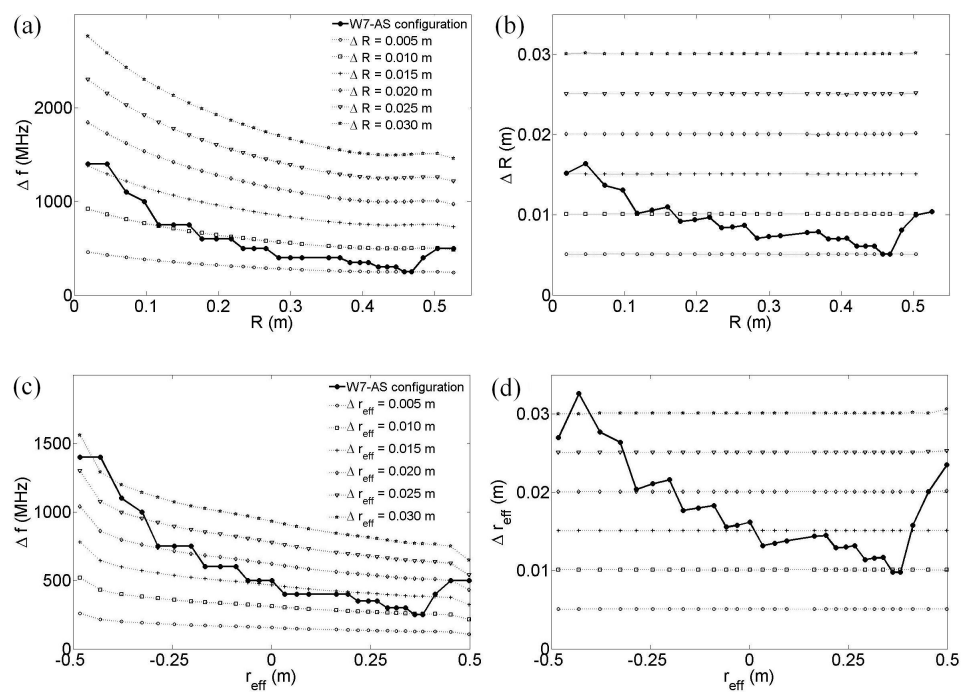

Figure 6: Filter bandwidths $\Delta \mathrm{f}$ (left) with resulting spatial resolution $\Delta \mathrm{R}$ and $\Delta \mathrm{r}_{e f f}$ (right) for chosen sight line of ECE diagnostic at Wendelstein 7-X. The upper row uses the laboratory coordinate $\mathrm{R}$ the lower one the so-called effective radius $\mathrm{r}_{e f f}$. The mapping from the laboratory coordinate $\mathrm{R}$ to the effective radius $\mathrm{r}_{e f f}$ enables the investigation of the radial resolution with respect to plasma Boozer coordinates. The position $\mathrm{R}=0 \mathrm{~m}\left(\mathrm{r}_{e f f}=-\right.$ $0.51 \mathrm{~m}$ ) marks the crossing of the sight line with the separatrix at the high-field side (inner torus side). The magnetic axis is located at $\mathrm{R}=0.267 \mathrm{~m}\left(\mathrm{r}_{e f f}=0 \mathrm{~m}\right)$. The crossing of the separatrix with the sight line at the low-field side is given by $\mathrm{R}=0.53 \mathrm{~m}\left(\mathrm{r}_{e f f}=0.51 \mathrm{~m}\right)$. The thick black line mimics the Wendelstein 7-AS configuration of the IF-device. The dashed lines show the bandwidth characteristics desired for a given spatial resolution of $5 \mathrm{~mm}$ to $30 \mathrm{~mm}$ measured in laboratory and effective coordinate respectively. The gap at $\mathrm{R}=0.33 \mathrm{~m}\left(\mathrm{r}_{e f f}=0.12 \mathrm{~m}\right)$ corresponds to the frequency of $140 \mathrm{GHz}$ blocked by the notch filter (figure $5 \mathrm{a}$ ). (a) $\Delta \mathrm{f}$ vs $\mathrm{R}$. (b) $\Delta \mathrm{R}$ vs. R. (c) $\Delta \mathrm{f}$ vs. $\mathrm{r}_{e f f}$. (d) $\Delta \mathrm{r}_{\text {eff }}$ vs. $\mathrm{r}_{\text {eff }}$. 
The application of the front-end in the ECE diagnostic at Wendelstein 7-X requires no changes. However, the IF-device has to be re-engineered. Since for the ECE diagnostic the frequency is connected with the radial position via the magnetic field, the spatial resolution can be improved by customizing the filter bandwidths in the filterbank. This requires the knowledge of the magnitude of the magnetic field along the chosen sight line as obtained from the software (see section 2.3). The resulting spatial resolution for the filter bandwidths can be evaluated for different spatial coordinates like the laboratory coordinate $\mathrm{R}$ and the corresponding effective radius $\mathrm{r}_{\text {eff }}$ from the so-called Boozer coordinates shown in figure 6 . Vice versa the combination of the bandwidths can be evaluated for a required spatial resolution (dashed lines in figure 6). The comparison shows that for the existent combination of the filter bandwidth the spatial resolution still varies by a factor of 3 along the ECE sight line. Furthermore 3 channels with the lowest center frequencies correspond to positions outside the last closed flux surface. Thus using the already existing equipment the temperature profile could only be measured at 29 radial positions. However, the remaining channels (here three) are important in studies of the electron energy distribution function indicating relativistically down-shifted emission of supra-thermal electrons localized mainly in the ECRH deposition region. More information about the distribution function is gained from ECE observation from the high-field side along the same sight line (chapter 2.1).

\section{Summary and outlook}

In this paper criteria for the design of the ECE sight line were discussed and the basic design for in-vessel optics has been introduced. A finite element analysis on the impact of the radiative heatload - up to $100 \mathrm{~kW} / \mathrm{m}^{2}$ under high performance steady state plasma conditions - to the corresponding plasma facing optical components is underway. Construction of the in-vessel optics is scheduled to start end 2008. Investigation of the ECE from suprathermal electrons requires an additional antenna viewing the plasma at the same line of sight but from the inner vessel, i.e. the high-field side. Such an antenna which must be integrated in the heat shield of the first wall is now designed. Further studies to extend the existing optics to a microwave imaging system have started. The 32 channel radiometer system already used at Wendelstein 7 -AS will be adapted to conditions at Wendelstein 7-X. In particular the radial resolution can be optimized to its physical limits determined by the relativistic broadening of the thermal ECE emission. After the assembly of the in-vessel optics and the radiometer an overall calibration experiment using the hot-cold source method is planned for mid 2009. This integral characterization of the ECE system as well as the careful study of its component characteristics will result in a description of the whole diagnostic by forward functions including an error analysis. The procedure ultimately aims on the capability to perform a probabilistic analysis of the ECE diagnostic and to obtain a quantitative assertion on the derived electron temperature profile information and its error sources $[7,8]$.

\section{Acknowledgements}

The authors want to thank A. Werner, Y. Turkin, D. Dodt, M. Krychowiak, N. Marushchenko and K. Ewert for their support. One of the authors (S.S.) is supported within the framework of the European Fusion Training Scheme as a trainee of the project Microwave Diagnostics Engineering in preparation for ITER. 


\section{References}

[1] Marushchenko N B et al 2006, Optimization of ECE diagnostic for Wendelstein 7-X stellarator, Fusion Sci. Technol. 50(3) 395-402

[2] Hartfuss H J et al 1997, Diagnostic strategy of the Wendelstein 7-X stellarator, Rev. Sci. Instrum. 68(2) 1244-1249

[3] Marushchenko N B et al, Analysis of ECCD scenarios for the Wendelstein 7-X Stellarator by high-field-side ECE measurements, 2004 31st EPS Conference on Plasma Phys., London

[4] Fuchs Ch, H J Hartfuss 2001, Extreme broadband multichannel ECE radiometer with "zoom" device, Rev. Sci. Instr. 72(1) 383-386

[5] Fuchs Ch, H-J Hartfuss 2001, Technology of the new Wendelstein 7-AS broadband radiometer system Fusion Eng. Des. 53 451-456

[6] Harftuss H J, Geist T, Hirsch M, PPCF 391693 (1997)

[7] Fischer R, A Dinklage and E Pasch, Bayesian modelling of fusion diagnostics, PPCF 45 1095$1111(2003)$

[8] Fischer R, C Wendland, A Dinklage, S Gori, V Dose and the W7-AS team, Thomson scattering analysis with the Bayesian probability theory, PPCF 44 1501-1519 (2002) 